\title{
Gene expression of growth signaling pathways is up-regulated in CD133-positive medulloblastoma cells
}

\author{
CHUNYU GU $^{1}$, NAOKI YOKOTA ${ }^{1,2}$, YUN GAO ${ }^{1}$, JUNKOH YAMAMOTO ${ }^{1}$, \\ TSUTOMU TOKUYAMA ${ }^{1}$ and HIROKI NAMBA ${ }^{1}$ \\ ${ }^{1}$ Department of Neurosurgery, Hamamatsu University School of Medicine, Higashi-ku, \\ Hamamatsu 431-3192; ${ }^{2}$ Yokohama CyberKnife Center, Asahi-ku, Yokohama 241-0014, Japan
}

Received November 5, 2010; Accepted December 30, 2010

DOI: $10.3892 / 01.2011 .235$

\begin{abstract}
Medulloblastoma (MB) is the most common malignant brain tumor in children. Cancer initiating cells (CICs) have been proposed to be involved in the development of brain tumors including MB. Prominin-1 antigen (CD133) is a candidate surface molecular marker for CICs. In the present study, CD133-positive cells were isolated from human Daoy $\mathrm{MB}$ cells and their gene expression was compared with that of control Daoy cells. DNA microarray analysis revealed that there were 398 up-regulated genes ( $>2$-fold increase) and 318 down-regulated genes $(<50 \%$ decrease) in the CD133positive cell-enriched fractions. Up-regulated genes included neuregulin-1, cyclin D1, cyclin-dependent kinase 6, vascular endothelial growth factor, inhibin $\beta \mathrm{A}$, promyelocytic leukemia gene, MYC, and hairy enhancer of split-1, which are components of growth signaling pathways. Molecular studies suggest that developmentally regulated signals important for stem cell maintenance are also involved in MB tumorigenesis. Moreover, these molecules can serve as novel targets for MB treatment.
\end{abstract}

\section{Introduction}

Medulloblastoma (MB) is the most common malignant brain tumor in childhood and is thought to arise from precursor cells in the cerebellar granule cell lineage (1). MB patients are now divided into stratification groups according to age, degree of resection and disease dissemination, and are treated depending upon risk. Although the use of multidisciplinary approaches and stratification management of the disease have improved prognosis, $50 \%$ of patients, particularly in the high-risk group, experience disease recurrence, dissemination to the cerebrospinal fluid space, and/or a high incidence of sequelae (2).

Correspondence to: Dr Hiroki Namba, Department of Neurosurgery, Hamamatsu University School of Medicine, 1-20-1 Handayama, Higashi-ku, Hamamatsu 431-3192, Japan

E-mail: hnamba@hama-med.ac.jp

Key words: medulloblastoma, cancer initiating cells, prominin-1 antigen, transcript analysis
The concept regarding the existence of cancer stem cells or cancer initiating cells (CICs) is currently a focal point. The hypothesis that cancerous cells originate from rare populations of CICs that are more tumorigenic than other cancer cells has gained increasing credence (3). CICs are thought to persist in tumors as a distinct population that can cause tumor recurrence and distant metastasis. The existence of CICs in $\mathrm{MB}$ has also been reported (4,5). Prominin-1 antigen (CD133) was identified in hematopoietic stem cells $(6,7)$ and neuroepithelial stem cells (8) and has generally been used as a marker for CICs (9). Although some investigators assert that CD133 is not an adequate marker of CICs since both CD133-positive and -negative cells are able to initiate tumors (10), it is also true that CIC-like cells that exhibit self-renewal and multipotential properties are restricted in the CD133-positive cell fractions. In the present study, CD133-positive cells were isolated from the human Daoy MB cell line using magnetic-activated cell sorting (MACS) beads and the transcript profiles of CD133-positive Daoy MB cells were investigated using DNA microarray analysis in order to obtain a better understanding of the molecular properties of CICs involved in MB tumorigenesis.

\section{Materials and methods}

Cell culture. The human Daoy medulloblastoma (MB) cell line was purchased from the American Type Culture Collection (ATCC) and cultured in Dulbecco's modified Eagle's medium (DMEM; Sigma-Aldrich, Inc., St. Louis, MO, USA) with $10 \%$ fetal bovine serum (FBS; Sigma-Aldrich, Inc.), $100 \mathrm{U} / \mathrm{ml}$ penicillin, and $100 \mu \mathrm{g} / \mathrm{ml}$ streptomycin at $37^{\circ} \mathrm{C}$ under $5 \% \mathrm{CO}_{2}$.

Flow cytometry. Cells were detached in phosphate-buffered saline (PBS) containing $0.25 \%$ trypsin and $0.02 \%$ EDTA for 3 min at $37^{\circ} \mathrm{C}$ under $5 \% \mathrm{CO}_{2}$, and the reaction was stopped by adding complete medium (DMEM with $10 \%$ FBS). Following centrifugation at $1000 \mathrm{rpm}$ for $5 \mathrm{~min}$, the cells were washed and resuspended in bovine serum albumin (BSA)/PBS buffer (PBS with $0.1 \%$ BSA and $2 \mathrm{mM}$ EDTA). Half of the cells were incubated with FcR blocking reagent (Miltenyi Biotec Inc., Auburn, CA, USA) and anti-CD133-PE (Miltenyi Biotec Inc.) for $10 \mathrm{~min}$ at $4^{\circ} \mathrm{C}$, and the remaining cells were incubated with IgG-PE (BD Biosciences, San Jose, CA, USA) as controls. After washing, the cells were resuspended in BSA/PBS buffer 
Table I. RT-PCR primer sequences of the genes of interest.

\begin{tabular}{llll}
\hline Gene symbol & Genebank & \multicolumn{1}{c}{ Sense } & \multicolumn{1}{c}{ Antisense } \\
\hline NRG1 & NM013959 & TTGGTGCTGCTTTCTTGTTG & CGGAGCCTCACACACCTATT \\
CCND1 & BC000076 & TCCTCTCCAAATGCCAGAG & TGAGGCGGTAGTAGGACAGG \\
CDK6 & NM001259 & AGGGTGCAGTCAAAACAACC & TCCCATCCACTTCAAAGGAG \\
VEGF & AF091352 & TGCAGATTATGCGGATCAAA & GCGAGTCTGTGTTTTGCAG \\
INHBA & M1343 & AGACGCTGCACTTCGAGATT & CCCTTTAAGCCCACTTCCTC \\
JAG1 & U73936 & AGCTGGCTTACACTGGCAAT & AAGTGGGAGCTCAAAGACCA \\
MYC & NM002467 & CTCCTGGCAAAAGGTCAGAG & TCGGTTGTTGCTGATCTGTC \\
HES1 & NM005524.2 & CTCTCTTCCCTCCGGACTCT & AGGCGCAATCCAATATGAAC \\
PML & AF230411 & GCAGCAGTGAGTCCAGTGA & GCTCTGCCTGCACTTCTTT \\
NFASC & NM015090.2 & TGCCTTGCTTTTGAGGAGAT & GGCTGTGGTCAGGGAAACTA \\
APOE & NM000041 & CCAATCACAGGCAGGAAGAT & AGCGCAGGTAATCCCAAAAG \\
ASTN & AB0006627 & ACAACACCCTCCTGGATCTG & AAGGAGTCCATTGCACCAAC \\
BMP2 & NM001200 & GGAGAATGCCCTTTTCCTCT & ACAACCCTCCACAACCATGT \\
NEFL & NM006158.2 & TCTGTTTGCTTGCAGAGTGG & GCTAACCACCGAAGGTTCAA \\
MAP2 & U89330 & AAGAAGGTCGCCATCATACG & GGCGGATGTTCTTCAGAGAG \\
GAPDH & & TGCACCACCAACTGCTTAG & GAGGCAGGGATGATGTTC \\
\hline
\end{tabular}

and analyzed using the Beckman Coulter Epics XL system (Beckman Coulter, Inc., Chaska, MN, USA). The data were analyzed using FlowJo software (Tree Star Inc., Ashland, OR, USA).

Cell sorting. CD133-positive Daoy cells were sorted using the CD133 cell isolation kit (Miltenyi Biotec Inc.). Briefly, cells were suspended in BSA/PBS buffer, incubated with FcR blocking reagent and CD133 microbeads (Miltenyi Biotec Inc.) for $30 \mathrm{~min}$ at $4^{\circ} \mathrm{C}$. To determine the sorting efficiency, the cells were incubated with anti-CD133/2-PE for $10 \mathrm{~min}$. Following washing and centrifugation, the cells were resuspended in BSA/PBS buffer, loaded onto a magnetic separation column (Miltenyi Biotec Inc.) and placed in a magnetic cell separator. The column was rinsed, and the magnetically labeled cells were flushed out with elution buffer and collected. These cells were used in the subsequent experiments.

DNA microarray analysis. Total RNAs were isolated from the CD133-positive Daoy cells (sorted and control) using TRIzol $^{\mathrm{TM}}$ (Invitrogen, Carlsbad, CA, USA). Synthesis and labeling of cRNAs and hybridization of biotin-labeled cRNA probes to the Human Genome U133A 2.0 expression Chip arrays (Affymetrix, Santa Clara, CA, USA) were performed according to the manufacturer's protocol. The imaging screens were scanned and analyzed using the Affymetrix Microarray Suite and GeneSpring GX (Agilent Technologies, Santa Clara, CA, USA).

Semi-quantitative reverse-transcriptase polymerase chain reaction $(R T-P C R)$ analyses. Total RNAs were prepared and used as templates for cDNA synthesis with random hexanucleotide primers and SuperScript reverse transcriptase II (Invitrogen). Real-time PCR analyses were performed using a QuantiTect SYBR-Green PCR kit (Takara, Kyoto, Japan) and a
LightCycler System (Roche, Basel, Switzerland). The PCR primer sequences were determined using WWW primer tool, Primer3 (http://biotools.umassmed.edu/bioapps/primer3_www. cgi) (Table I). The transcript abundance of the genes of interest was normalized to that of glyceraldehyde-3-phosphate dehydrogenase (GAPDH) mRNA as an internal standard. At least 3 independent analyses were performed for each sample and for each gene.

\section{Results}

CD133-positive Daoy MB cells were highly enriched by $M A C S$. Flow cytometry showed that $3-5 \%$ of Daoy cells expressed prominin-1 antigen (CD133) and CD133/2 antigens. After MACS was applied, the CD133-positive cells were highly enriched $(>60 \%)$. These cells were then used in the DNA microarray gene expression analyses.

Transcript analysis in CD133-positive MB cells. Transcript analysis using DNA microarrays was performed, and the acquired data were filtered according to the gene expression level. In comparison with the control Daoy cells, the CD133positive cell-enriched fractions exhibited a $>2$-fold increase in the expression of 398 genes, and a $<50 \%$ decrease in the expression of 318 genes. A number of molecules involved in the growth signaling pathways, which play important roles both in $\mathrm{MB}$ oncogenesis and stem cell proliferation, were up-regulated in the CD133-positive cell-enriched fractions. These molecules included neuregulin-1 (NRG1; which showed a 6.818-fold increase), cyclin D1 (CCND1; 5.636), cyclin-dependent kinase 6 (CDK6; 3.564), vascular endothelial growth factor (VEGF; 3.186), inhibin $\beta$ A (INHBA; 3.115), Jagged 1 (JAG1; 2.702), promyelocytic leukemia gene (PML; 2.538), MYC (2.479), and hairy enhancer of split-1 (HES1; 2.078) (Table II). On the other hand, neural differentiation markers or developmentally 
Table I. Gene changes in the CD133-positive Daoy cells.

\begin{tabular}{|c|c|c|c|c|}
\hline Symbol & Genebank & Map & Fold change & Gene name \\
\hline \multicolumn{5}{|c|}{ Up-regulated genes } \\
\hline RGS16 & U94829 & $1 q 25-q 31$ & 10.87 & Regulator of G-protein signaling 16 \\
\hline NRG1 & NM_013959 & 8p21-p12 & 6.818 & Neuregulin 1; a ligand for the NEU/ERBB2 \\
\hline CCND1 & ВC000076 & $11 q 13$ & 5.636 & Cyclin D1 \\
\hline JUN & BC002646 & 1p32-p31 & 5.161 & V-jun sarcoma virus 17 oncogene homolog (avian) \\
\hline CASP2 & BC002427 & $7 q 34-q 35$ & 5.081 & Caspase 2, apoptosis-related cysteine peptidase \\
\hline EGR1 & NM_001964 & $5 q 31.1$ & 3.963 & Early growth response 1 \\
\hline MET & AA005141 & $7 q 31$ & 3.886 & Met proto-oncogene (hepatocyte growth factor receptor) \\
\hline Cep290 & AF317887 & $12 q 21.33$ & 3.883 & Centrosome protein cep290 \\
\hline CDK6 & NM_001259 & $7 q 21-q 22$ & 3.564 & Cyclin-dependent kinase 6 \\
\hline MAX & NM_002382 & $14 q 23$ & 3.531 & MYC associated factor X \\
\hline DKK1 & NM_012242 & $10 \mathrm{q} 11.2$ & 3.277 & Dickkopf homolog 1 (Xenopus laevis) \\
\hline VEGF & AF091352 & $6 \mathrm{p} 12$ & 3.186 & Vascular endothelial growth factor \\
\hline INHBA & M13436 & 7p15-p13 & 3.115 & Inhibin, $\beta \mathrm{A}$ (activin $\mathrm{A}$, activin $\mathrm{AB} \alpha$ polypeptide) \\
\hline PYGO1 & AL049925 & $15 q 21.1$ & 3.104 & Pygopus homolog 1 (Drosophila) \\
\hline JAG1 & U73936 & 20p12.1-p11.23 & 2.702 & Jagged 1 (Alagille syndrome) \\
\hline HDAC9 & NM_014707 & $7 \mathrm{p} 21.1$ & 2.651 & Histone deacetylase 9 \\
\hline KHSRP & AI933301 & $19 \mathrm{p} 13.3$ & 2.643 & KH-type splicing regulatory protein (FUSE binding protein 2) \\
\hline NPAT & U58852 & $11 q 22-q 23$ & 2.564 & Nuclear protein, ataxia-telangiectasia locus \\
\hline GADD45B & NM_015675 & $19 \mathrm{p} 13.3$ & 2.563 & Growth arrest and DNA-damage-inducible, $\beta$ \\
\hline PML & AF230411 & $15 q 22$ & 2.538 & Promyelocytic leukemia \\
\hline GREM1 & NM_013372 & $15 q 13-q 15$ & 2.508 & Gremlin 1, cysteine knot superfamily, homolog \\
\hline MYC & NM_002467 & 8q24.12-q24.13 & 2.479 & \\
\hline CCNT1 & NM_001240 & 12pter-qter & 2.41 & Cyclin T1 \\
\hline TGFBR1 & NM_004612 & $9 q 22$ & 2.408 & Transforming growth factor, $\beta$ receptor I \\
\hline EGFR & U95089 & $7 \mathrm{p} 12$ & 2.299 & Epidermal growth factor receptor \\
\hline CCNE2 & AF112857 & $8 q 22.1$ & 2.162 & Cyclin E2 \\
\hline SMAD5 & AF010601 & $5 q 31$ & 2.125 & SMAD, mothers against DPP homolog 5 (Drosophila) \\
\hline HES1 & BE973687 & $3 q 28-q 29$ & 2.078 & Hairy and enhancer of split 1 (Drosophila) \\
\hline SMAD3 & NM_005902 & $15 q 21-q 22$ & 2.016 & SMAD, mothers against DPP homolog 3 (Drosophila) \\
\hline \multicolumn{5}{|c|}{ Down-regulated genes } \\
\hline NFASC & AI821777 & - & 0.0608 & Neurofascin homolog (chicken) \\
\hline IGF1 & AI972496 & $12 q 22-q 23$ & 0.166 & Insulin-like growth factor 1 (somatomedin C) \\
\hline IGFBP5 & AW007532 & $2 q 33-q 36$ & 0.175 & Insulin-like growth factor binding protein 5 \\
\hline SEMA3E & NM_012431 & $7 q 21.11$ & 0.197 & Semaphorin 3E \\
\hline APOE & NM_000041 & $19 q 13.2$ & 0.296 & Apolipoprotein E \\
\hline $\mathrm{BBP}$ & AA012917 & $1 \mathrm{p} 32.1$ & 0.356 & TM2 domain containing 1 \\
\hline VCAM1 & NM_001078 & $1 \mathrm{p} 32-\mathrm{p} 31$ & 0.359 & Vascular cell adhesion molecule 1 \\
\hline SLIT3 & $\mathrm{AB} 011538$ & $5 q 35$ & 0.328 & Slit homolog 3 (Drosophila) \\
\hline RARRES2 & ВC000069 & $7 q 36.1$ & 0.365 & Retinoic acid receptor responder (tazarotene induced) 2 \\
\hline ASTN & $\mathrm{AB} 006627$ & $1 q 25.2$ & 0.392 & Astrotactin \\
\hline BMP2 & AA583044 & $20 \mathrm{p} 12$ & 0.397 & Bone morphogenetic protein 2 \\
\hline $\mathrm{TNC}$ & BF434846 & $9 q 33$ & 0.404 & Tenascin C (hexabrachion) \\
\hline UNC5B & AA127885 & $10 \mathrm{q} 22.2$ & 0.406 & Unc-5 homolog B (C.elegans) \\
\hline NEFL & AL537457 & 8p21 & 0.418 & Neurofilament, light polypeptide $68 \mathrm{kDa}$ \\
\hline RBP1 & NM_002899 & $3 q 23$ & 0.448 & Retinol binding protein 1 , cellular \\
\hline CDH11 & AU144378 & - & 0.459 & Cadherin 11 , type 2 , OB-cadherin (osteoblast) \\
\hline RAI16 & NM_022749 & $8 \mathrm{p} 21.3$ & 0.461 & Retinoic acid induced 16 \\
\hline CASP4 & AL050391 & $11 q 22.2-q 22.3$ & 0.48 & Caspase 4 , apoptosis-related cysteine peptidase \\
\hline MAP2 & U89330 & 2q34-q35 & 0.49 & Microtubule-associated protein 2 \\
\hline
\end{tabular}

The genes of interest are indicated in boldface type. 
A
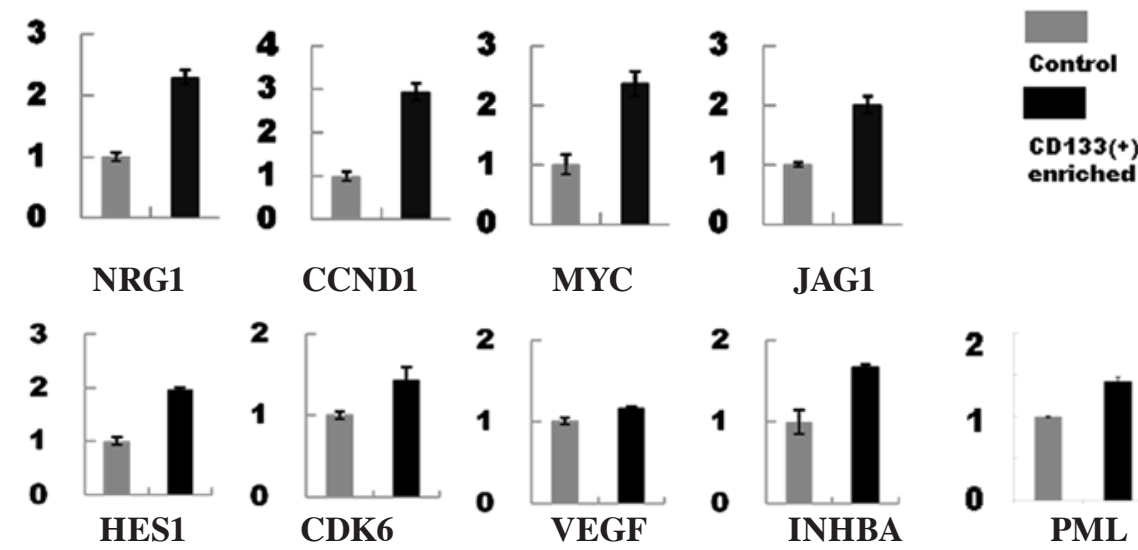

JAG1
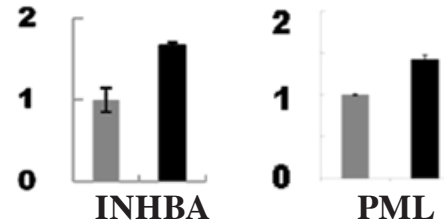

B

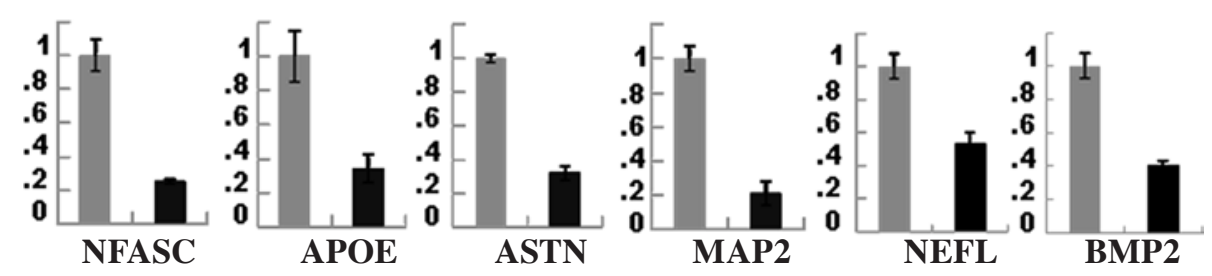

Figure 1. Expression analyses of the genes of interest using semi-quantitative RT-PCR analyses of the up-regulated (A) and down-regulated genes (B).

regulated genes, expressed in the granule cell lineage, such as neurofascin (NFASC; 0.0608), apolipoprotein E (APOE; 0.296), astrotactin (ASTN; 0.392), neurofilament light polypeptide $68 \mathrm{kDa}$ (NEFL; 0.418), and microtubule-associated protein 2 (MAP2; 0.49) were down-regulated (Table II). Semi-quantitative RT-PCR analyses were then performed in the selected genes (up-regulated genes, Fig. 1A; downregulated genes, Fig. 1B) and the gene expression changes were confirmed to be significant.

\section{Discussion}

In the present study, we first isolated CD133-positive cells in the human Daoy medulloblastoma (MB) cell line. The percentage of CD133-positive cells was approximately 3-5\%, which was in accordance with previous studies $(0.5-10 \%)(11,12)$. After MACS was applied, the percentage of CD133-positive cells was noted to be greater than $60 \%$. These enriched cell fractions were subsequently subjected to transcript analysis using DNA microarrays.

Transcript analysis using DNA microarrays identified various molecules that were components of the growth signaling pathways, which play important roles both in $\mathrm{MB}$ oncogenesis and stem cell proliferation. The genes which exhibited up-regulated expression included the activator of MAP kinase signal (RGS16), a ligand of ERBB (EGF signal component; NRG1), Wnt signal targets CCND1 and c-myc, a ligand of Notch signal (JAG1), and its target (HES1) (13-15) (Table II). c-myc is known to play a key role in stem cell selfrenewal and was used to produce induced pluripotent stem cells (16). The Wnt and Notch pathways are involved in the maintenance of stem cell properties and in MB oncogenesis (17-19). The remaining up-regulated genes included INHBA, an inhibitor of differentiation factors, such as activin and TGF $\beta$, and VEGF which plays a role in the neovascularization of tumors $(20,21)$. These genes may be involved in tumor recurrence or distant metastasis.

In contrast, the genes whose expression decreased to less than $50 \%$ included the neural markers (MAP2 and NEFL) $(22,23)$, developmentally regulated genes in the cerebellar granule cell lineage (NFASC, UNC5B, ASTAN, SLIT3 and APOE) (24-27), and molecules involved in retinoic acidinduced apoptosis in neuroblastoma (RBP1, BMP2, RARRES2 and CASP4) $(28,29)$. Down-regulation of these genes may result in the inhibition of differentiation and maintenance of undifferentiated properties of CICs or may contribute to the inhibition of cell death, thereby providing infertility to CICs.

An understanding of the molecular pathway involved in $\mathrm{MB}$ oncogenesis has been advanced by analyses of the Turcot- and Gorlin-inherited syndromes which are associated with the development of MB. The Wnt and sonic hedgehog ( $\mathrm{SHH})$ signal pathways are involved in $\mathrm{MB}$ oncogenesis in the Turcot and Gorlin syndromes, respectively (30-33). In addition, the Notch, epidermal growth factor receptor ERBB, and platelet-derived growth factor (PDGF) signaling pathways are involved in MB oncogenesis or prognosis (33-37). These pathways play crucial roles in the proliferation and/or differentiation of the cerebellar granule cell lineage where MB originates. Furthermore, molecular studies have shown that developmentally regulated signals, such as Wnt, $\mathrm{SHH}$ and Notch, play important roles in self-renewal, proliferation and/or the multipotency of stem cells, and are also involved in MB oncogenesis (17-19). These molecular studies and the results of the present study indicate that further understanding of the molecular properties and fundamental signaling pathways of CICs involved in MB oncogenesis may lead to the 
development of new, more effective, and less toxic treatment modalities for $\mathrm{MB}$, thereby improving the quality of life of children with MB.

\section{Acknowledgements}

We express sincere appreciation to Professor Y. Koide, Department of Microbiology and Immunology, Hamamatsu University School of Medicine, Professor T. Nagata, Dr S. Seto and Dr M. Uchijima and other members of Professor Koide's Laboratory for helpful advice, technical assistance and valuable discussions. This study was supported by a fund from the Japanese Ministry of Education, Culture, Sports, Science and Technology (no. 17501509).

\section{References}

1. Louis DN, Ohgaki H, Wiestler OD and Cavenee WK (eds) WHO Classification of Tumours of the Central Nervous System. 4th edition. International Agency for Research on Cancer, Lyon, 2007.

2. Crawford JR, MacDonald TJ and Packer RJ: Medulloblastoma in childhood: new biological advances. Lancet Neurol 6: 1073-1085, 2007.

3. Clarke MF and Fuller M: Stem cells and cancer: two faces of eve. Cell 124: 1111-1115, 2006

4. Singh SK, Hawkins C, Clarke ID, et al: Identification of human brain tumour initiating cells. Nature 432: 396-401, 2004.

5. Hemmati HD, Nakano I, Lazareff JA, et al: Cancerous stem cells can arise from pediatric brain tumors. Proc Natl Acad Sci USA 100: 15178-15183, 2003

6. Miraglia S, Godfrey W, Yin AH, et al: A novel five-transmembrane hematopoietic stem cell antigen: isolation, characterization, and molecular cloning. Blood 90: 5013-5021, 1997.

7. Yu Y, Flint A, Dvorin EL and Bischoff J: AC133-2, a novel isoform of human $\mathrm{AC} 133$ stem cell antigen. J Biol Chem 277: 20711-20716, 2002.

8. Fargeas CA, Corbeil D and Huttner WB: AC133 antigen, CD133, prominin-1, prominin-2, etc.: prominin family gene products in need of a rational nomenclature. Stem Cells 21: 506-508, 2003.

9. Mizrak D, Brittan M and Alison MR: CD133: molecule of the moment. J Pathol 214: 3-9, 2008.

10. Shmelkov SV, Butler JM, Hooper AT, et al: CD133 expression is not restricted to stem cells, and both $\mathrm{CD} 133^{+}$and $\mathrm{CD} 133$ metastatic colon cancer cells initiate tumors. J Clin Invest 118 2111-2120, 2008

11. Blazek ER, Foutch JL and Maki G: Daoy medulloblastoma cells that express CD133 are radioresistant relative to CD133- cells, and the $\mathrm{CD} 133^{+}$sector is enlarged by hypoxia. Int $\mathrm{J}$ Radiat Oncol Biol Phys 67: 1-5, 2007.

12. Srivastava VK and Nalbantoglu J: Flow cytometric characterization of the DAOY medulloblastoma cell line for the cancer stem-like phenotype. Cytometry A 73: 940-948, 2008.

13. Buckbinder L, Velasco-Miguel S, Chen Y, et al: The p53 tumor suppressor targets a novel regulator of $\mathrm{G}$ protein signaling. Proc Natl Acad Sci USA 94: 7868-7872, 1997.

14. Guarnaccia C, Pintar A and Pongor S: Exon 6 of human Jagged-1 encodes an autonomously folding unit. FEBS Lett 574: 156-160, 2004.

15. Gilbertson RJ, Clifford SC, MacMeekin W, et al: Expression of the ErbB-neuregulin signaling network during human cerebellar development: implications for the biology of medulloblastoma. Cancer Res 58: 3932-3941, 1998.
16. Takahashi K, Tanabe K, Ohnuki M, et al: Induction of pluripotent stem cells from adult human fibroblasts by defined factors. Cell 131: 861-872, 2007.

17. Reya $\mathrm{T}$ and Clevers $\mathrm{H}$ : Wnt signalling in stem cells and cancer. Nature 434: 843-850, 2005.

18. Ruiz i Altaba A, Sanchez P and Dahmane N: Gli and hedgehog in cancer: tumours, embryos and stem cells. Nat Rev Cancer 2: 361-372, 2002

19. Gilbertson RJ: Medulloblastoma: signalling a change in treatment. Lancet Oncol 5: 209-218, 2004.

20. Brown CW, Houston-Hawkins DE, Woodruff TK and Matzuk MM: Insertion of Inhbb into the Inhba locus rescues the Inhba-null phenotype and reveals new activin functions. Nat Genet 25: 453-457, 2000.

21. Holash J, Maisonpierre PC, Compton D, et al: Vessel cooption, regression, and growth in tumors mediated by angiopoietins and VEGF. Science 284: 1994-1998, 1999.

22. Garner CC, Tucker RP and Matus A: Selective localization of messenger RNA for cytoskeletal protein MAP2 in dendrites. Nature 336: 674-677, 1988.

23. Julien JP, Grosveld F, Yazdanbaksh K, Flavell D, Meijer D and Mushynski W: The structure of a human neurofilament gene (NF-L): a unique exon-intron organization in the intermediate filament gene family. Biochim Biophys Acta 909: 10-20, 1987.

24. Ango F, di Cristo G, Higashiyama H, Bennett V, Wu P and Huang ZJ: Ankyrin-based subcellular gradient of neurofascin, an immunoglobulin family protein, directs GABAergic innervation at purkinje axon initial segment. Cell 119: 257-272, 2004.

25 . Wingate RJ: The rhombic lip and early cerebellar development. Curr Opin Neurobiol 11: 82-88, 2001.

26. Yuan W, Zhou L, Chen JH, Wu JY, Rao Y and Ornitz DM: The mouse SLIT family: secreted ligands for ROBO expressed in patterns that suggest a role in morphogenesis and axon guidance. Dev Biol 212: 290-306, 1999.

27. Lafarga M, Crespo P, Berciano MT, Andres MA and Leon J: Apolipoprotein $\mathrm{E}$ expression in the cerebellum of normal and hypercholesterolemic rabbits. Brain Res Mol Brain Res 21: 115-123, 1994.

28. Hallahan AR, Pritchard JI, Chandraratna RA, et al: BMP-2 mediates retinoid-induced apoptosis in medulloblastoma cells through a paracrine effect. Nat Med 9: 1033-1038, 2003.

29. Gumireddy K, Sutton LN, Phillips PC and Reddy CD: All-transretinoic acid-induced apoptosis in human medulloblastoma: activation of caspase-3/poly(ADP-ribose) polymerase 1 pathway. Clin Cancer Res 9: 4052-4059, 2003.

30. Goodrich LV, Milenkovic L, Higgins KM and Scott MP: Altered neural cell fates and medulloblastoma in mouse patched mutants. Science 277: 1109-1113, 1997.

31. Wechsler-Reya R and Scott MP: The developmental biology of brain tumors. Annu Rev Neurosci 24: 385-428, 2001.

32. Yokota N, Nishizawa S, Ohta S, et al: Role of Wnt pathway in medulloblastoma oncogenesis. Int J Cancer 101: 198-201, 2002.

33. Yokota N, Mainprize TG, Taylor MD, et al: Identification of differentially expressed and developmentally regulated genes in medulloblastoma using suppression subtraction hybridization. Oncogene 23: 3444-3453, 2004.

34. Fan X, Matsui W, Khaki L, et al: Notch pathway inhibition depletes stem-like cells and blocks engraftment in embryonal brain tumors. Cancer Res 66: 7445-7452, 2006

35. Gilbertson R, Wickramasinghe C, Hernan R, et al: Clinical and molecular stratification of disease risk in medulloblastoma. Br J Cancer 85: 705-712, 2001

36. MacDonald TJ, Brown KM, LaFleur B, et al: Expression profiling of medulloblastoma: PDGFRA and the RAS/MAPK pathway as therapeutic targets for metastatic disease. Nat Genet 29: 143-152, 2001

37. Gilbertson RJ and Clifford SC: PDGFRB is overexpressed in metastatic medulloblastoma. Nat Genet 35: 197-198, 2003. 